\title{
Le marketing, ou la ruse de l'économie
}

Résumé. L'article s'interroge sur la contribution des marketers et du marketing au fonctionnement de l'économie de marché. Il montre comment, au début du siècle, les économistes déviants qui ont fondé la discipline sont parvenus à jeter des ponts entre science et maîtrise des marchés, d'abord en détachant progressivement les savoir-faire pratiques des managers, puis en retraduisant ces savoirs sous la forme d'une science de gestion orientée vers la compréhension et la maîtrise des marchés concrets. Dans l'entre deux guerres, l'application du taylorisme industriel à la gestion des marchés et l'arrimage de la science commerciale au volontarisme industriel du New Deal ont permis au marketing d' "avoir prise » sur le cours des choses économiques, et de reprendre en «sous-main invisible» le jeu d'un marché défaillant. Les mutations ultérieures du marketing sous la forme d'une science positive, d'un académisme pur, voire d'une science sociale souvent hostile au monde de la gestion n'ont paradoxalement fait qu'étendre les catégories et les outils de la discipline à d'autres compartiments de la société et de l'économie, au point que l'on peut aujourd'hui se demander si le marketing n'est pas devenu, avec le temps, la ruse de l'économie.

\begin{abstract}
The paper discusses the possible contribution of marketers and marketing to the functioning of the market economy. Through the gathering of the managers' various forms of know-how and the reformulation of them as a science of real markets, the deviant economists who pioneered the marketing field at the beginning of the century succeeded in building bridges between the science of markets and their practice. In the interwar period, the application of industrial taylorism to the management of markets and the junction between marketing and the New Deal helped marketing to get a hold on the economy and to clandestinely animate the game of a weakened market. Paradoxically enough, the later transformations of marketing as a positive science, as pure academism or even as a social science often hostile towards managerial interests have only but extended the categories and the tools of the discipline to other compartments of society and economy. This evolution leads us to wonder if today marketing has not become the hidden motive of modern economics.
\end{abstract}

\author{
Franck Cochoy \\ Université Toulouse II/CERTOP, UMR CNRS 5044
}

Maison de la Recherche, 5 allées Antonio Machado, 31058 Toulouse CEDEX

E-mail : cochoy@univ-tlse2.fr 
Grâce à toute une série de circonstances [...] on a trouvé le moyen de détacher [les] expériences de chacune des entreprises dans lesquelles elles ont été acquises, de les réunir, de les conserver et de les transmettre comme un patrimoine objectivé. C'est cette formidable masse d'expérience qui permet, lorsqu'elle est utilisée, de pousser le rationalisme économique au plus haut degré de perfection. ${ }^{1}$

Comment fonctionne l'économie de marché ? D'un côté les économistes orthodoxes soutiennent depuis longtemps que l'équilibre des marchés repose sur le jeu libre de l'offre et de la demande; d'un autre côté les économistes hétérodoxes, mais aussi les historiens, les sociologues et les anthropologues du marché se sont efforcés, chacun à sa manière, de montrer combien l'offre et la demande sont socialement construites, combien la pratique gestionnaire détermine les contours des marchés. Paradoxalement, si nous renouvelons ici ce constat, c'est moins pour radicaliser l'opposition classique des deux camps que pour souligner la base commune de leur opposition : d'une part, les discussions des uns et des autres partent toujours de l'offre et/ou de la demande, de leur caractère plus ou moins «naturel » ou contingent ; d'autre part leurs propos distinguent toujours la science (économique) et la pratique (gestionnaire), afin d'examiner l'adéquation problématique de l'une à l'autre.

Mettre en cause les fondements de cette discussion sans fin nous semble le moyen d'en sortir. Pour comprendre l'économie de marché, il suffit en effet de regarder ailleurs, de poser d'autres questions : le fonctionnement de l'économie de marché ne repose-t-il pas sur d'autres instances que l'offre et la demande? Le fonctionnement des marchés ne dépend-il pas d'autres procédures que celles de la science économique et/ou de la pratique gestionnaire? En posant ces questions, on découvre qu'il existe, entre l'offre et la demande, tout une série d'acteurs - courtiers, marchands, représentants, consultants, experts, professeurs - qui contribuent à façonner l'une et l'autre. On s'aperçoit qu'il existe, entre la science économique et la pratique gestionnaire, l'ensemble méconnu des sciences de gestion, ces «sciences de la pratique » ou ces «pratiques instrumentées par la science » qui concourent pourtant à mettre en œuvre l'une et l'autre.

Allons plus loin. Parmi les sciences de gestion, parmi les tiers qui animent le marché, les marketers méritent sans doute un examen particulier. Nous voudrions montrer combien les médiateurs marchands se sont employés, tout au long de l'histoire du capitalisme moderne, à occuper la position centrale : entre producteurs et consommateurs, à mi-chemin de la science économique et de la pratique managériale, les spécialistes du marketing sont peu à peu

1. Sombart (W.), Le bourgeois, Contribution à l'histoire morale et intellectuelle de l'homme économique moderne, Paris, Payot, p. 329. 
parvenus à réinventer les acteurs et les processus fondamentaux de l'échange économique, à discipliner (maîtriser/codifier) l'économie de marché, à la «performer ». De même que, chez le linguiste Austin, un énoncé performatif est un énoncé qui dit et fait ce qu'il dit simultanément (par exemple : «je déclare la séance ouverte») ${ }^{2}$. Michel Callon et Bruno Latour ont montré qu'une science performative est une science qui décrit et qui construit son objet en même temps ${ }^{3}$. Ici, nous reprendrons cette proposition, en montrant plus précisément que la progressive performation de l'économie par le marketing a revêtu quatre aspects successifs : d'abord, les pionniers du marketing se sont donné pour objectif de se former à l'étude empirique du marché et de former des spécialistes correspondants. Les marketers sont parvenus à ce premier objectif en formatant le savoir de la pratique via la constitution de cadres conceptuels et humains adéquats. À partir de là, les adeptes de la discipline du marché ont joué le jeu des gestionnaires et des affaires, des économistes et de l'économie pour finalement redéfinir les contours du marché et de l'économie dans leur ensemble ${ }^{4}$.

\section{La formation des pairs : le double apprentissage du marketing}

Bien sûr la discipline du marketing (le contrôle des marchés) a commencé bien avant la discipline du marketing (la science des marchés). De ce point de vue, la mise en lumière du marketing comme élément de structuration des marchés n'a rien d'innovant. Les historiens américains ont depuis longtemps montré que les savoir-faire des gestionnaires en matière commerciale ont participé de façon décisive à l'affirmation du capitalisme contemporain : depuis le milieu du 19e siècle, l'internalisation progressive du marché dans les grandes entreprises $^{5}$, la pratique récurrente de la segmentation des clientèles ${ }^{6}$, voire la construction sociale de la demande ${ }^{7}$ ont sanctionné la prise de pouvoir de la main visible des managers au

2. Austin (J.L.), How to Do Things With Words, London, Oxford University Press, 1962.

3. Callon (M.) \& Latour (B.), «"Tu ne calculeras pas” ou comment symétriser le don et le capital », Revue du MAUSS semestrielle, $\mathrm{n}^{\circ}$ 9, premier semestre, Paris, La Découverte, 1997.

4. Le présent article est une version française, remaniée et mise à jour, d'un texte initialement publié en anglais : Cochoy (F.), «Another Discipline for the Market Economy: Marketing as a Performative Knowledge and Know-how for Capitalism », in The Laws of the Markets, Callon (M.) (ed.), Sociological Review Monographs Series, Oxford, Blackwell, 1998, pp. 194-221. Je remercie Michel Callon pour m'avoir donné l'occasion d'écrire ce texte et de le présenter lors de l'atelier consacré à la discussion du livre The laws of the markets à la London School of Economics. Je remercie aussi Luc Boltanski, David Courpasson, Éric Godelier et Michel Grossetti pour m'avoir permis d'en débattre dans le cadre des séminaires qu'ils ont organisé, respectivement, à l'EHESS, à l'E-M. Lyon, à l'IAE de Poitiers et à l'Université Toulouse II. Les remarques des organisateurs, discutants et participants de ces divers séminaires m'ont été très utiles. Bien sûr, les propos tenus dans l'article n'engagent que moi-même.

5. Cochran (Th.C.) 200 Years of American Business, New York, Basic Books, 1977 ; Chandler (A.D. Jr.), La main visible des managers : une analyse historique, Paris, Economica, 1988 [1977].

6. Tedlow, (R.S.), New and Improved: The Story of Mass Marketing in America, New York, Basic Books, 1990.

7. McKendrick (N.), Brewer (J.) \& Plumb (J.H.), The Birth of a Consumer Society. Bloomington, Indiana University Press, 1982 ; Mukerji (Ch.), From Graven Images : Patterns of Modern Materialism, New York, 
détriment de la main invisible du marché. Tant que le marketing se confond avec l'offre, tant que la technique commerciale est un simple prolongement de l'action de l'entreprise et des entrepreneurs, sa mise à jour ne fait que renforcer l'idée d'une construction sociale des marchés, d'une fabrication de l'économie théorique par les acteurs économiques classiques : le producteur et le consommateur.

Pourtant, l'histoire du marketing est aussi celle de la progressive dissociation de la connaissance et de la pratique marchandes. Or, nous voudrions montrer que la constitution du marketing comme corps de savoir distinct, situé à mi-chemin entre l'offre et la demande, entre les enjeux de la pratique et les procédures de la science ${ }^{8}$, change tout: l'émergence du marketing comme discipline (règle/référence) de l'économie de marché permet la circulation des savoirs, la formation de connaissances et d'hommes nouveaux, donc la mise en oeuvre d'idées et de pratiques susceptibles de renouveler non seulement l'identité des acteurs en présence, mais surtout d'infléchir le cours même des choses économiques.

\section{De l'économie au marketing :découvrir le marketing}

Pour comprendre comment l'affirmation du marketing comme discipline de gestion a contribué au fonctionnement des marchés, il n'est pas anodin d'observer combien, au départ, le marketing universitaire provient de la science économique elle-même. Les fondateurs du marketing universitaire moderne furent des économistes du Middle West américain, formés à l'école de l'économie historique allemande. Ce furent des économistes, parce que l'économie était la seule discipline qui, à l'époque, traitait des phénomènes marchands ${ }^{9}$. Ce furent des économistes américains formés en Allemagne, parce que la formation de ceux-là les opposait aux orientations classiques de leurs pairs formés aux États-Unis, parce que l'école historique allemande dont ils étaient issus prônait une approche des choses économiques qui fût à la fois historique, statistique, et pragmatique, c'est-à-dire tournée vers l'étude empirique des marchés concrets $^{10}$. Enfin, ce furent des économistes du Middle West, parce que ces personnes, souvent membres de Land Grant Universities, confrontées quotidiennement aux problèmes complexes de l'économie agricole, furent amenées à s'intéresser au fonctionnement réel des

Columbia University Press, 1983 ; Campbell (C.), The Romantic Ethic and the Spirit of Modern Consumerism, New York, Basic Blackwell, 1987 ; Strasser (S.), Satisfaction Guaranteed: The Making of the American Mass Market, New York, Pantheon Books, 1989.

8. Pour davantage de précisions sur la position du marketing comme science, $c f$. Cochoy (F.), « Les sciences du social et leur demande : le cas du marketing », Sciences de la société, numéro spécial Production scientifique et demande sociale, $\mathrm{n}^{\circ} 49$, février 2000, pp. 47-61.

9. Bartels (R.), The History of Marketing Thought (2nd ed.), Columbus, Grid, 1976.

10. Jones (D.G.B.) \& Monieson (D.D.), «Early Development of the Philosophy of Marketing Thought», Journal of Marketing, Vol. 54, January 1990, pp. 102-113. 
circuits de distribution, et notamment à l'acheminement des denrées périssables des zones rurales vers les zones urbaines ${ }^{11}$.

À l'écart de l'économie politique classique, mais aussi à l'extérieur du monde de l'entreprise dont il ne connaissaient rien, les économistes fondateurs du marketing durent tout inventer. Cette extrême marginalité fit leur force. Pour construire un savoir nouveau, ces hommes entreprirent de suivre physiquement le parcours des produits tout au long des circuits de distribution et d'inventorier les institutions, procédures et pratiques observées. Le témoignage de L.D.H. Weld, auteur du tout premier manuel de marketing ${ }^{12}$, est à cet égard exemplaire :

Lorsque j'ai commencé à enseigner le marketing à l'automne 1913, il n'y avait pratiquement pas de littérature sur le sujet. Je devais aller sur le terrain et déterrer ma propre information. J'ai étudié de première main le mouvement du grain et le fonctionnement du marché à terme de la Chambre de Commerce de Minneapolis [...] J'ai suivi personnellement l'envoi par bateau du beurre et des œufs et d'autres marchandises depuis l'affréteur de campagne du Minnesota en passant par les grossistes, intermédiaires et détaillants jusqu'à New York, Chicago, et autres villes. J'ai analysé chaque dépense impliquée dans ce passage à travers les circuits du commerce. J'ai étudié les méthodes de cotation des prix, les opérations d'échange du beurre et des œufs, et les marchés d'enchères des villes de l'Est. J'ai aussi étudié de première main les opérations des associations coopératives de fret du Minnesota et publié des bulletins sur ce sujet. ${ }^{13}$

Parce qu'il n'est de nulle part, parce qu'il ne sait rien, le futur professeur de marketing passe au site de l'autre, en prélève le savoir. En visitant l'ensemble des lieux, il construit un savoir transversal. Avant lui, le savoir existait, mais retranché en chaque point de la chaîne. Après lui, le savoir est extériorisé, intégré, redistribué. On obtient enfin l'image d'ensemble :

Après deux ans de ce travail j'avais écrit mon livre, «Le Marketing des Produits Agricoles ». ${ }^{14}$

L'universitaire ne sait rien, donc, et lorsqu'il apprend quelque chose, il fait profession de le faire deux fois : il enseigne ce qu'il a récolté. Dès lors, ce hors-jeu, cet intrus dans la concurrence historique des acteurs privés, construit sa propre médiation, propose la diffusion transversale de savoirs détournés, resitués, restitués, anticipe une possible performation de l'économie par la nouvelle science gestionnaire.

11. Converse (P.D), The Beginning of Marketing Thought in the United States with Reminiscences of the Pioneer Scholars, Studies in Marketing, No. 3, Austin, The University of Texas, Bureau of Business Research, 1959.

12. Weld (L.D.H.), The Marketing of Farm Products, New York, McMillan, 1916.

13. Weld (L.D.H.), «Early Experiences in Teaching Courses in Marketing », Journal of Marketing, Vol. 5, April 1941, pp. 380-381.

14. Ibid. 


\section{Des pratiques aux cours de marketing : enseigner le marketing}

Mais pour performer l'économie de marché, le marketer devait encore devenir le formateur des marchands. Or, au départ, cela n'avait rien d'évident : pourquoi les hommes d'affaires auraient-ils prêté attention aux leçons de professeurs qui, jusqu'à preuve du contraire, en savaient moins qu'eux-mêmes ? Pourquoi auraient-ils renoncé à se former sur le tas, par la pratique? Pourquoi auraient-ils admis l'inventaire et la divulgation de leurs précieux savoirs, au risque de perdre leur expertise et leurs avantages concurrentiels ? Pourquoi auraient-ils troqué un savoir local mais éprouvé contre un savoir général mais mal assuré ?

Répondre à cette quadruple question, c'est d'abord observer que l'invention du marketing universitaire est contemporaine d'une double mise en réseau. Du côté des entreprises, au début du siècle, on assistait à la formation du groupe des cadres. À cette époque, ceux qui assuraient la gestion technique des entreprises se rencontraient, se rassemblaient, organisaient des meetings, lançaient des revues professionnelles. Ces efforts aboutirent en 1919 à la formation d'une American Administrative Management Association qui, en 1925, devint l'American Management Association. Parallèlement, la formation universitaire se généralisait et s'unifiait: l'Association of American Universities fut créée en 1900, l'American Association of University Professors en 1915, l'American Council on Education en 1918.

L'entreprise grandissait, et le management devenait une profession - celle des cadres. L'université se développait, et la formation supérieure devenait une profession - celle des universitaires. La professionnalisation des uns était indissociable de celle des autres. Les universités avaient pour mission de prendre en charge les qualifications, d'assurer l'homogénéisation des critères de formation et des carrières. Seules les institutions d'enseignement se trouvaient en mesure de mettre au point les diplômes qui garantiraient les compétences d'acteurs anonymes entrant sur le marché du travail nouveau des spécialistes en gestion. En retour, les cadres, qui n'avaient d'autres richesses que leur unique savoir-faire, étaient les seuls, dans le monde de l'entreprise, à être directement intéressés à la constitution des sciences propres à légitimer leur action, à garantir leur emploi, à construire leur identité commune face au groupe des actionnaires ${ }^{15}$.

Voilà pourquoi les business schools se développèrent rapidement, et s'unirent, en 1916, au sein d'une American Association of Collegiate Schools of Business. Les écoles de gestion furent le point de ralliement des mouvements conjoints d'affirmation des cadres et d'essor des universités. Ce furent des lieux de formation, si l'on veut bien entendre par ce mot autant

15. Pour davantage de précisions, $c f$. Cochoy (F.), « Savoir des affaires et marché du travail : la naissance des disciplines de gestion à Northwestern University », Genèses, n 34 , mars 1999, pp. 80-103.. 
formation des savoirs que formation des savants, autant formation des professionnels que formation des professeurs. Les business schools, comme leur nom l'indique, permirent le brassage des affaires et du scolaire, donc la redéfinition de l'identité de chacun des bords ainsi rapprochés. À ce titre, il n'est pas innocent de rappeler combien les pionniers du marketing furent des hommes-hybrides, croisant et combinant allègrement les identités de gestionnaire et d'universitaire, passant de l'université à l'entreprise, puis de l'entreprise à l'université (etc.).

Les premiers (Ralph Starr Butler, L.D.H. Weld, Paul H. Nystrom...), économistes déviants devenus marketers, parvinrent, en alternant les positions universitaires et gestionnaires, à faire en sorte que le savoir empirique en matière de gestion des marchés gagnât en importance, en reconnaissance, pour finir par intégrer le centre, les lieux de savoir et de pouvoir des régions, des universités (NYU, Columbia...) et des entreprises les plus importantes de l'Amérique (Procter \& Gamble, U.S. Rubber...). Ces hommes purent ainsi, telle l'abeille qui transporte le pollen récolté d'une fleur à l'autre, fertiliser chacun des champs traversés par les savoirs acquis dans les autres.

Les seconds, en migrant temporairement de l'entreprise vers l'université, purent obtenir une position suffisamment décalée et surplombante pour penser l'articulation des affaires. Arch W. Shaw en particulier, homme d'affaires ayant des intérêts dans plusieurs entreprises de Chicago, et par-là même porté à comparer des situations diverses pour constituer un savoir transversal des affaires, fut requis successivement par Harvard et Northwestern University pour contribuer au développement de cursus en gestion. Arch W. Shaw tira parti des effets de perspective ouverts par la multiplicité de ses positions pour inventer les références, les dispositifs et les méthodes grâce auxquels on pourrait unifier la nouvelle communauté des spécialistes en gestion.

Du côté des affaires, Arch W. Shaw fonda System, the Magazine of Business - l'ancêtre direct de Business Week —, c'est-à-dire un forum de discussion, un lieu où les hommes de l'art pouvaient découvrir des idées générales sur la conduite des affaires, partager et mettre à l'épreuve leurs savoirs, convictions et expériences communes. Du côté de l'université, Arch W. Shaw fut à l'origine de deux innovations capitales. D'une part, il mit en place la forme laboratoire pour l'entreprise - le bureau of business research ${ }^{16}$ - ce dispositif universitaire prodigieux qui, en ouvrant sur la constitution d'un savoir à la fois systématique, cumulatif et transversal, rendait pour la première fois, aux yeux des praticiens, l'expertise des universitaires supérieure à leur propre expérience individuelle. D'autre part, il mit au point la méthode des cas, cette didactique novatrice qui, en transposant la pratique dans la salle de

16. Arch W. Shaw mit en place de tels centres de recherche d'abord à Harvard, puis à Northwestern University. 
classe, donnait pour la première fois aux enseignants le privilège d'une pédagogie de la pratique, mais permettait aussi, en organisant la simulation de la pratique, l'acquisition de l'expérience (le savoir) par le recours à l'expérience (l'expérimentation), d'apprendre la pratique sans les risques du métier.

Tous ces premiers efforts de généralisation et de croisement des savoirs et des pratiques locales ne tardèrent pas à susciter l'apparition de procédures globales de mise en forme et de mise en équivalence, tant la circulation des hommes en économie de marché - et notamment l'invention de possibilités de carrières pour les cadres - demandait l'uniformisation des langages, des qualifications, et des compétences. En marketing, cette uniformisation emprunta une double voie.

\section{Le marketing comme pré-formatage : discipliner l'économie}

\section{La première uniformisation du marketing : la sédimentation d'un savoir commun}

La première voie d'uniformisation du marketing, ascendante et interactive, fut celle de la mise en relation progressive des observations et des connaissances acquises sur des terrains locaux en matière de produits, d'institutions et de fonctions commerciales. Nous avions vu plus haut que pour percer les mystères des circuits de distribution, l'économiste-marketer avait choisi de suivre la commercialisation des produits. Le suivi des produits déboucha sur la nomination de leurs lieux d'origine, de transit, et de destination : dans les années 1910-1920, les pères fondateurs du marketing moderne, formés pour la plupart, nous l'avons vu, à l'école de l'économie institutionnelle, entreprirent de décrire l'ensemble des institutions impliquées dans le processus de commercialisation, depuis les maisons de gros jusqu'au moindre commerce de détail. À partir de ce double inventaire (ce qui circule : les produits ; par où cela circule : les institutions) une première généralisation devint possible. La double entrée par les produits et par les institutions déboucha sur l'approche fonctionnelle, à l'époque où, précisément, la «fonction marketing» commençait à être institutionnalisée dans les entreprises américaines ${ }^{17}$.

L'étude des «fonctions » de marketing fut introduite par Arch W. Shaw, qui. proposa une classification des «fonctions générales des intermédiaires ${ }^{18}$. Les fonctions qu'il distinguait

17. Faria (A.J.), «The Development of the Functional Approach to the Study of Marketing to 1940 », in Hollander (S.C) \& Savitt (R.) (eds.), First North American Workshop on Historical Research in Marketing, East Lansing, Michigan State University, 1983, pp. 160-169.

18. Shaw (A.W.), «Some Problems in Market Distribution», Quarterly Journal of Economics, Vol. 26, 
étaient le partage des risques, le transport, le financement, la vente, l'assemblage-assortimentréexpédition. L'idée d'Arch W. Shaw était que ces fonctions, universelles, devaient être remplies d'une façon ou d'une autre : si quelque chose pouvait être éliminé dans la médiation marchande, c'était tel ou tel intermédiaire, mais non la fonction que ce dernier remplissait. Parce qu'elle dissociait les hommes, les choses et les concepts, l'approche fonctionnelle ouvrait sur la généralisation, donc sur la sédimentation transversale des savoirs locaux. Cette approche fut reprise par l'ensemble des premiers manuels qui s'efforcèrent de compléter, d'affiner, de systématiser la typologie initiale d'Arch W. Shaw, par exemple en regroupant les fonctions initialement discernées sous des intitulés plus généraux ${ }^{19}$.

Ainsi, peu à peu, la connaissance des réseaux déboucha sur un réseau de connaissances ; l'induction pragmatique allant des produits aux institutions puis des institutions aux fonctions accoucha d'une discipline et d'un corps de spécialistes entièrement nouveaux. Comme en témoigne un historien-marketer, jadis étudiant auprès des pères fondateurs, et excellent sociologue :

le premier problème (i.e. définir le champ et le mode d'accès au champ) fut résolu à l'époque par ladite approche par produit, fonction, agences-institutions [...] c'était, pour des chercheurs universitaires et leurs étudiants, une façon acceptable, pragmatique, d'entrer dans cette contrée relativement inexplorée. Aussi curieux que cela puisse paraître aujourd'hui, cela faisait sens! Pourquoi ? Parce que cette approche fournissait un cadre d'analyse auquel le professeur comme les étudiants pouvaient rapporter leurs propres formation, expérience et environnement — et ils le firent avec énergie $!^{20}$

L'uniformisation progressive des connaissances par lectures réciproques, rapprochements et recoupements conduisit les hommes de marketing à se repérer les uns les autres, à dialoguer, à se regrouper. Ainsi, l'uniformisation ascendante et décentralisée des connaissances (au sens de savoirs) dont nous parlions plus haut fut très vite relayée par l'uniformisation descendante et centralisée des connaissances (au sens de relations humaines), que nous allons évoquer maintenant.

August 1912, pp. 703-765.

19. Ainsi, Frederick E. Clark regroupait sous le triptyque «Fonctions d'échange», «Fonctions d'offre physique », «Fonctions auxiliaires ou de facilitation» les sous-fonctions (respectivement) de création de demande et d'assemblage ; de transport et de stockage ; de financement, de prise de risque et de standardisation Clark (F.E.), Principles of Marketing, New York, Macmillan, 1922, cité in Faria (A.J.), « The Development of the Functional Approach to the Study of Marketing to 1940 », op. cit., pp. 164-165.

20. Grether (E.T.), «Macro-Micro Marketing in Perspective», in Shapiro (S.) \& Walle (A.H.) (eds.), Marketing, A Return to the Broader Dimensions, Chicago, American Marketing Association, 1988, p. iv. 


\section{La seconde uniformisation du marketing : la constitution d'une communauté humaine}

Les universitaires intéressés par le monde commercial se rencontrèrent dans le cadre des associations professionnelles de leurs origines — Associated Advertising Clubs of the World pour les psychologues marginaux parmi les professionnels de la publicité naissante, American Economic Association pour les économistes marginaux parmi les économistes orthodoxes. À l'occasion de ces réunions, les marginaux des deux bords prirent d'abord conscience de leur marginalité dans leur communauté d'origine, et se regroupèrent. Les deux groupes ainsi formés, en raison de leur intérêt commun pour la distribution et de leur identité commune d'universitaires déviants, finirent par fusionner en une National Association of Teachers of Marketing and Advertising ${ }^{21}$. Enfin, ce premier groupe fut bientôt rejoint par un troisième : le groupe des cadres et des techniciens spécialistes de l'étude qui, s'estimant en marge du pouvoir gestionnaire et du savoir universitaire, s'étaient regroupés depuis 1931 en une American Marketing Society. Les hommes de l'entre-deux décidèrent donc de se joindre aux autres hommes de l'entre-deux, et de donner naissance, le premier janvier 1937, à l'American Marketing Association ${ }^{22}$.

L'apparition d'une association professionnelle ad hoc permit aux hommes de marketing d'accroître leur emprise sur la formation et sur la gestion de l'économie américaine. Nous avions signalé que la mise en œuvre des nouveaux savoirs-pratiques dépendait de leur généralisation, de leur abstraction, de leur systématisation sous forme d'enseignement. Cependant, cette première procédure d'uniformisation ascendante, de par son caractère itératif et décentralisé, était lente et capricieuse ; elle reposait sur des rencontres aléatoires, sur des trouvailles providentielles, sur la disponibilité plus ou moins grande des références, sur le bon vouloir et la perspicacité des auteurs comme des éditeurs. Une association professionnelle, en revanche, permettait de lever ces difficultés. Dès les années 1920, l'ancêtre de l'AMA, la National Association of Teachers of Advertising - mit en place un Committee on Teachers' Materials destiné à rassembler du matériel pédagogique auprès des publicitaires et des éditeurs, puis elle se lança dans sa propre activité éditoriale, en publiant les communications de son colloque annuel sous forme de bulletins ${ }^{23}$. Rassembler des textes et les diffuser permettait à la communauté concernée d'accéder simultanément au même type de références : les marketers, par l'intermédiaire des écrits, pouvaient pour la première fois communiquer, prendre connaissance de l'action des autres, rapporter leurs positions respectives aux avancées de leurs autres collègues.

21. Agnew (H.E.), «The History of the American Marketing Association », Journal of Marketing, Vol. 5, April 1941, pp. 376-377.

22. Ibid., p. 375.

23. Ibid. 
Le rapprochement était enfin possible, mais il suscitait plus de problèmes qu'il n'en résolvait. En effet, la mise à jour des travaux divers faisait apparaître en pleine lumière l'hétérogénéité des entreprises locales, la prolifération des concepts, l'extrême polysémie des incarnations possibles du «marketing », tour à tour synonyme de vente, de distribution, de publicité, etc. On pouvait comparer, certes, mais la comparaison tendait à produire une impression de cacophonie préjudiciable à la cohésion intellectuelle et sociale de l'association. Aussi, le deuxième effort des responsables de l'association consista à mettre de l'ordre dans les éléments qu'elle avait contribué à rassembler, à construire un langage commun.

En 1930 fut institué un Committee on Definitions qui reçut pour mission de légiférer, d'harmoniser, de construire le vocabulaire officiel du marketing. Parce que l'AMA occupait une position centrale, elle pouvait niveler puis redistribuer le sens des mots et des choses, constituer un dictionnaire. Parce que les acteurs — membres et non-membres — n'avaient pas que cela à faire, et trouvaient au contraire commode que quelqu'un se chargeât à leur place de définir le langage qui leur permettrait de mieux communiquer, donc d'aller plus vite dans la conduite de leurs échanges et de leurs projets personnels, ils se rangèrent volontiers, de proche en proche, aux définitions promues par l'AMA ; ils échangèrent les objections qu'ils auraient pu émettre s'ils avaient participé directement à la négociation des contours du marketing contre le gain d'énergie et de temps que leur offrait justement la délégation du problème d'uniformisation.

Mais l'objectif des marketers n'était pas uniquement de produire des mots et des concepts, c'était surtout de faire en sorte que les notions élaborées fussent reprises et incorporées dans les pratiques. Pour que les définitions formulées par l'AMA ne restassent pas lettres mortes, il fallait donc montrer comment ces outils pouvaient jouer ensemble ; il convenait de passer du paradigme au syntagme; il s'agissait d'articuler le vocabulaire en un tout cohérent, utilisable par ses éventuels locuteurs. En même temps, comme l'intérêt pour les études de marché allait croissant, et comme les membres de l'AMA n'étaient pas, loin de là, les seuls à en maîtriser la pratique, mieux valait tenter de rester dans la course, en répertoriant et en intégrant le savoir accumulé ici et là dans un ensemble logique. À cet effet, en 1937, fut institué un Committee on Marketing Techniques, qui publia aussitôt un ouvrage de synthèse ${ }^{24}$. Grâce à cet ouvrage, l'AMA pouvait consolider son rôle d'instance-référence, et productrice de références, pour l'ensemble de la discipline et de ses clientèles.

Pourtant, les responsables de l'AMA, en bons spécialistes du marketing, savaient bien que toute prétention au monopole est illusoire, et que ni le langage, ni le savoir, ne peuvent être définitivement fixés. Comment concilier la référence (le point de repère fixe) et le 
renouvellement des références (la mouvance permanente des connaissances) ? Pour assurer leur position de référence, les hommes de l'AMA devaient mobiliser un dispositif qui assurât à la fois la permanence de leur identité disciplinaire et institutionnelle et la révision continue des acquis de la discipline. Ce moyen, ce fut le Journal of Marketing. La revue scientifique est à la fois toujours et jamais la même. Son titre invariant permet de fédérer une communauté, mais son contenu fluctuant permet aussi d'enregistrer fidèlement les déplacements, les mouvements, les mutations qui l'affectent.

Ainsi, dans les premières années de sa parution, le Journal of Marketing refléta fidèlement la généalogie conceptuelle de la discipline. Un bilan des 10 premières années de publication de la revue montrait bien que les thématiques originelles (marketing agricole, relations entre théories économiques et marketing, enseignement du marketing...) avaient progressivement cédé le pas à des orientations nouvelles, telle la gestion des ventes ou, surtout, les études de marchée ${ }^{25}$. En d'autres termes, les préoccupations initiales visant à fonder conceptuellement la nouvelle discipline, à faire le tour des pratiques de marketing, à trouver les méthodes adéquates de son enseignement — bref, l'entreprise visant à partir de l'extérieur pour percer le savoir interne de l'entreprise - avaient progressivement débouché sur la maîtrise de la gestion technique des relations entre l'entreprise et son marché.

Le glissement thématique des publications, mais aussi l'unification et la généralisation du vocabulaire et des savoir-faire, la multiplication des lexiques, des répertoires, des manuels, des revues, la mise en place des institutions, des meetings et des cercles humains, bref, le préformatage progressif de la pratique marketing par les savoirs et par les réseaux du même nom attestaient la mise en place d'un bouclage de plus en plus étroit des savoirs et de la pratique, l'application œuvre des concepts sous forme de cadres d'actions disciplinaires et gestionnaires. Désormais, par la double action de l'enseignement (local) et de l'harmonisation (globale) de la nouvelle discipline, la cognition se repliait sur la gestion ; l'étroite imbrication d'un corpus professoral et d'un corps professionnel parvenait peu à peu à pénétrer le monde commercial, à le faire agir sinon de concert, du moins dans un même état d'esprit, et donc à peser sur l'orientation générale de l'économie américaine, tant au niveau micro que macroéconomique.

25. Applebaum (W.), «The Journal of Marketing: The First Ten Years », Journal of Marketing, Vol. 11, 1947 , p. 356. 


\section{La performance du marketing : jouer dans et avec l'économie}

\section{Une performance microéconomique : le marketing taylorien}

Au niveau microéconomique, la mise au pas du marketing américain autour d'un esprit et d'un bagage technique communs fut préparée et prolongée par l'arrimage progressif de la science commerciale aux préceptes d'un taylorisme en pleine expansion. Puisque la «gestion scientifique» du travail permettait au management d'obtenir un meilleur contrôle de l'organisation de la production, certains pensèrent que le modèle pouvait être transposé et adapté au monde commercial pour avoir prise sur le système de distribution.

D'abord, dès 1912, un certain Charles W. Hoyt s'employa à tayloriser la vente comme Taylor avait taylorisé l'atelier: chez Hoyt, la fixation de quotas de vente, l'établissement d'itinéraires précis et l'imposition d'une formation professionnelle standardisée devenaient aux vendeurs ce que l'assignation des rendements, la définition des tâches et la planification des procédures étaient au travailleur de Taylor :

Dans la Gestion Scientifique de la Force de Vente, je mettrais fortement l'accent sur une division plus égale des responsabilités entre le représentant et la direction. [...] Pour ce faire, je créerais un service central de planification chargé des vendeurs. [...] La Gestion Scientifique de la Force de Vente a également trait à la sélection appropriée de tel homme pour telle tâche. [...] Le directeur des ventes qui travaille sous les principes de la Gestion Scientifique de la Force de Vente réalise que sa tâche est de former systématiquement cet homme que l'on désire compétent, plutôt que de pister celui que quelqu'un d'autre a formé. ${ }^{26}$.

Pour la première fois, la déclinaison d'un programme de «gestion scientifique de la vente » permettait d'étendre la «gestion scientifique du travail » à l'extérieur de l'atelier, de généraliser l'optimisation et le contrôle des activités gestionnaires à l'extérieur de la firme.

Ensuite, en 1927, un dénommé Percival White s'appliqua à radicaliser et systématiser ce programme. Hoyt avait étendu le contrôle taylorien de la production vers la vente ; White proposa d'inverser la relation, de partir d'une "gestion scientifique des marchés » pour contrôler la production dans son ensemble. Préfigurant le marketing concept et le marketing management des années 1950 - lesquels, nous le verrons, entendent subordonner la gestion de l'entreprise à la satisfaction préalable du consommateur - White proposa de soumettre l'ensemble du processus productif à la maîtrise scientifique non seulement de la vente, mais aussi de la distribution dans son ensemble :

La guerre a depuis longtemps été déclarée contre les pertes de production. Cependant, ces pertes sont d'une importance secondaire. Avant qu'elles puissent être éliminées, les pertes de marketing doivent être réduites, parce que le marketing est premier et que toute la production dépend à terme de la demande du

26. Hoyt (Ch.W.), Scientific Sales Management, New Haven, George B. Woolson \& Co., 1912, pp. 25-26. 
marché. Les méthodes de fabrication de masse, avec la gestion scientifique des hommes et des machines, peuvent réussir à réduire le coût de la production, mais les économies réalisées de cette façon sont d'une certaines manière neutralisées en raison du désordre de la distribution, de la concurrence destructrice, et de procédures de marketing inorganisées. [...] Les gaspillages dus à une diversification inutile sont éliminés par un marketing scientifique. Les études de marché indiquent les attentes de la consommation, à la fois présentes et potentielles. ${ }^{27}$

Avec le marketing taylorien, le taylorisme du dedans finissait par être encadré par un taylorisme du dehors ; d'un bout à l'autre du marché américain, de la production jusqu'à la consommation, ou plutôt de la consommation jusqu'à la production, le marché américain se trouvait enserré, défini, géré, optimisé par l'application d'une même doctrine, d'une même procédure, d'un même contrôle ${ }^{28}$.

Entre 1912 et 1927, des énoncés de Hoyt aux formulations de White, en arrière du basculement d'une simple gestion des ventes vers une gestion inspirée par l'observation et le contrôle méthodiques du marché, il y avait eu, bien sûr, la progression des savoirs, des lexiques, des techniques, des associations, des réseaux et des institutions du marketing. Certes, le marketing taylorien des uns et le marketing plus descriptif des autres ne se superposaient pas. Mais les deux formes de marketing avançaient parallèlement, se renforçaient l'une l'autre, et permettaient d'appliquer à la gestion des entreprises américaines tout un ensemble de concepts et d'instruments d'après lesquels il devenait enfin possible de ne plus concevoir chaque entreprise comme plongée dans le marché, mais au contraire de situer le marché au cœur même de la gestion de toute entreprise. Avec le marketing, la boucle de la gestion scientifique était bouclée : tout le circuit économique, de l'entreprise jusqu'au marché, devenait passible d'un contrôle systématique - le marketing passait progressivement de la microéconomie à la macroéconomie.

\section{Une performance macroéconomique : le marketing du New Deal}

À ce stade de notre exposé et de ce point de vue, il n'est pas anodin d'observer combien la proposition du marketing comme moyen d'adjoindre à la gestion de l'entreprise une gestion symétrique du marché coïncide avec un soudain accès de faiblesse de l'autorégulation marchande. L'essor décisif du marketing, comme science et comme technique de gestion est contemporain de la crise de 1929 : dans les années 1930, les marketers s'emparèrent d'un domaine de recherche inédit — la régulation gouvernementale du marketing - et leur communauté fut rejointe par des membres d'un nouveau type - les fonctionnaires de l'État

27 White, (P.), Scientific Marketing Management: Its Principles and Methods, New York, Harper \& Bros, 1927.

28. Cochoy (F.), «La gestion scientifique des marchés : marketing et taylorisme dans l'entre-deux-guerres », Recherche et Applications en Marketing, Vol. 9, n² 2, 1994, pp. 97-114. 
Fédéral. Quelques indices le montrent: au cours des années de crise, dans les pages du Journal of Marketing, les préoccupations gouvernementales avaient conquis la deuxième place $(16,7 \%$ des articles), alors que les agents de l'État représentaient la troisième force humaine de l'American Marketing Association (15,1\% des membres) ${ }^{29}$. Que s'était-il passé ?

Pour nous, qui connaissons l'incidence finale de la crise sur l'économie américaine, la réponse est facile : la crise économique des années 1930 était passée par là, le contexte avait produit son effet, les problèmes de l'époque s'étaient retrouvés naturellement inscrits dans le programme de l'Association ${ }^{30}$. Marc Meuleau peut écrire — en exagérant quelque peu — « le marketing va trouver [dans la crise de 1929] son acte de naissance $»^{31}$. Mais pour les acteurs de l'époque, les choses n'étaient pas aussi évidentes. Dans les années 1930, ce n'était pas le contexte qui imprimait son effet sur les acteurs, mais bien plutôt les acteurs qui se saisissaient du contexte pour assurer leur propre progression. De ce point de vue, le tout premier numéro de The American Marketing Journal, publié en 1934, contenait un avertissement de ses rédacteurs fort explicite :

$[1]^{32}$ Nous réalisons tous que les problèmes extrêmement importants auxquels les entreprises auront à faire face au cours de la prochaine décennie et même au-delà tomberont presque certainement dans le domaine du marketing et de la distribution. [2] L'efficacité de nos processus productifs a apparemment surclassé les développements correspondants du marketing. [...] [3] Alors que ce premier numéro part sous presse, la National Recovery Administration et l'Agricultural Adjustment Administration font des efforts audacieux pour hâter le retour de la prospérité. [4] Quels que puissent être leur succès et leurs réalisations ultimes, ils auront certainement produit une impression durable sur la pensée des affaires. [5] Face à la spirale infernale descendante de la dépression, les hommes d'affaires auront pu développer un cadre de pensée qui les rende désireux d'accepter le leadership selon des conditions qu'ils n'auraient pas voulu considérer quelques années auparavant. [6] L'Administration pense que la stabilité de l'emploi et des salaires adéquats sont de la première importance pour fournir un marché de masse à notre production de masse, et la nation est unie dans un grand effort concret visant à donner à cette conception une mise en œuvre universelle. [7] Les résultats seront étroitement observés par les cadres du marketing, qui consacreront une attention croissante aux données et aux horaires comme indices des possibilités de vente. [...] [8] Le but de The American Journal est tout d'abord de présenter un matériau valable qui sera intéressant pour ceux qui ont la charge des opérations de marketing dans les sociétés d'affaires. [9] En d'autres termes, nous espérons être un facteur susceptible d'aider à vendre les résultats de véritables études de marché aux responsables de la gestion. [10] L'un des problèmes avec la plupart des recherches

29. Applebaum (W.), «The Journal of Marketing : The First Ten Years », op. cit., pp. 356-357.

30. Par exemple, le tout premier numéro de The American Marketing Journal (l'un des deux ancêtres du Journal of Marketing), contenait les articles suivants : «Conditions présentes sous la N.R.A. (National Recovery Administration)» (Edward P. Warner) ; « Nouvelles conditions de marketing sous le code du commerce de détail (Retail Code)» (Paul H. Nystrom); «Les impacts possibles de la N.R.A. et de la A.A.A. (Agricultural Adjustment Administration) sur le marketing des produits alimentaires» (V.H. Peltz), etc. (in The American Marketing Journal, A Journal for the Advancement of Science in Marketing, Vol. 1, No. 1, January 1934).

31. Meuleau (M.), «L'introduction du marketing dans l'entreprise en France (1880-1973) », Revue Française de Gestion, Les racines de l'entreprise, Numéro spécial, $n^{\circ} 70$, septembre-octobre 1988, p. 62.

32. Entre crochets, nous attribuons un numéro à chacune des phrases auxquelles notre commentaire fera référence. 
en gestion est que leurs résultats pratiques sont rarement présentés aux responsables d'une façon qui les rende propres à être utilisés pour modifier les méthodes d'achat et de vente des biens ${ }^{33}$.

La dépression, (parce que) désastreuse pour les affaires, était une opportunité fantastique pour les nouveaux spécialistes du marketing. Le chaos économique déstabilisait les certitudes des anciens gestionnaires, et les rendait par conséquent plus réceptifs à la mise en œuvre de principes de gestion nouveaux. Bien entendu, pour les nouveaux marketers, la crise ne valait que si ses effets profitaient à la nouvelle expertise. Voilà pourquoi on prenait soin, d'une part d'associer désordre économique et problèmes distributifs [1], et d'autre part de disqualifier les savoirs existants [2]. «Vos problèmes sont des problèmes de marketing, et vous ne pourrez plus les résoudre par vous-mêmes » : voilà comment les marketers présentaient la crise aux managers - leurs clients [9]. L'action du gouvernement Roosevelt, ici, donnait aux marketers un ultime argument. Non seulement les conditions économiques avaient changé, mais les principes de gestion n'étaient plus les mêmes [3]. À la régulation marchande se substituait désormais l'interventionnisme fédéral, avec toute sa batterie de codes, de règlements, d'agences spécialisées, de dispositifs comptables, de nouveaux principes économiques [3]. Comme l'acceptation du New Deal était universelle [6], au point que la communauté des affaires elle-même semblait en accepter la fatalité $[4 ; 5]^{34}$, il fallait bien «faire avec», repenser la vie des affaires dans le cadre d'une économie faisant une place plus large à l'intervention de l'État [7]. Tel était précisément le programme du marketing: la nouvelle discipline prenait acte de la nouvelle donne, formulait des concepts adaptés à ses effets, offrait en retour aux entreprises les moyens d'un interventionnisme microéconomique [8]. Le Journal, dès lors, se chargerait de mettre en forme ces moyens, et de les communiquer à ses destinataires [10].

Le New Deal, de contexte macroéconomique avec majuscules, devenait «new deal », complexe microsocial avec minuscules. Le projet de l'État fédéral servait à formuler celui d'une discipline nouvelle. À l'instar de l'État Providence moderne, le marketing se structurait au moment où tout s'effondrait ${ }^{35}$; le marketing tirait parti des ratés de la main invisible pour imposer sa propre médiation. Pour les conseillers du Prince comme pour ceux de l'entrepreneur, les soubresauts de l'économie libérale servaient de fondation à la construction des sciences nouvelles; les représentants des secteurs public et privé brandissaient le

33. The American Marketing Journal, op. cit., pp. 3-4.

34. L'affirmation, bien entendu, était plus rhétorique qu'empirique. Si l'État Fédéral parvint à soumettre en un an près de 600 activités à la régulation des codes prévus par le National Industrial Recovery Act, il se heurta immédiatement à l'opposition ferme et continue de la National Association of Manufacturers (regroupement du petit commerce et de la petite industrie), de sorte que le dispositif officiel finit par échouer (Cochran (Th.C.), Business in American Life: A History, New York, McGraw-Hill, 1972, pp. 318-319).

35. Fullerton (R.A.), « Modern Western Marketing as a Historical Phenomenon : Theory and Illustration », in Nevett (T.) \& Fullerton (R.) (eds.), Historical Perspectives in Marketing: Essays in Honor of Stanley C. Hollander, Lexington, Lexington Books, 1988, p. 83. 
spectacle des effets de l'absence d'une science économique adéquate pour justifier l'émergence de la macroéconomie d'un côté, du marketing de l'autre. Des deux côtés, on proposait une reprise en main générale du marché - la main visible du manager et la main prévoyante de l'État devaient pouvoir, en unissant leurs efforts, contrôler définitivement les fluctuations économiques.

Le parallélisme des deux projets mérite d'être souligné. Alors que les marketers tentaient de s'organiser, en promouvant la régulation de l'AMA sur leur profession, l'administration Roosevelt s'efforçait de maîtriser l'économie, en imposant la régulation de l'État sur le cours des choses économiques. D'un côté, les marketers défendaient les études de marché comme moyen de répondre aux attentes précises de la clientèle. D'un autre côté, l'État fédéral prenait fait et cause pour la normalisation industrielle comme outil susceptible d'accroître la lisibilité du marché ${ }^{36}$. L'élaboration de codes industriels était particulièrement conforme à la pensée marketing: si l'État visait à la construction de conventions $^{37}$ propres à permettre une meilleure identification des produits, les marketers voyaient dans le même procédé une arme stratégique pour la conquête d'avantages concurrentiels : l'État ouvrait la porte au contrôle technique de l'extension de l'accord marchand, l'élaboration des conventions (niveau global) devenait l'outil privilégié de la différenciation et de la segmentation (niveau local).

En outre, le dirigisme étatique - la prise en main de l'économie - apportait une légitimation d'une ampleur inégalée aux études de marchés. De l'étude des marchés locaux, l'État passait à l'étude $d u$ marché global: il donnait une application et une caution universelles à ce qui, jusqu'à présent, n'avait été qu'une pratique locale et particulière. Ainsi, le lancement en 1929 du premier recensement national de la distribution ${ }^{38}$ justifiait non

36. Nous faisons ici référence aux « codes de concurrence loyale » élaborés conjointement, dans le cadre du National Industrial Recovery Act de 1933, par les représentants des industriels, de l'État et des travailleurs. Ces chartes fixant pour chaque secteur concerné les prix et les conditions de travail étaient censés lutter contre la concurrence déloyale - la vente à perte — , donc enrayer la chute des prix et l'accroissement du chômage. Les industriels qui avaient accepté de coopérer recevaient le droit d'imprimer sur leur produit un aigle bleu, label auquel les consommateurs étaient invités à donner leur préférence. Cet astucieux dispositif fut toutefois déclaré illégal par la Cour Suprême en 1936.

37. Au sens de Luc Boltanski et Laurent Thévenot (Boltanski (L.) \& Thévenot (L), De la justification. Les économies de la grandeur, Paris, Gallimard, 1991).

38. À partir du milieu des années 1920, les bureaucrates de Washington, désireux d'alimenter l'appareil d'État en données et de promouvoir leur expertise bureaucratique tant à l'intérieur qu'à l'extérieur de l'administration s'intéressèrent au recueil de statistiques commerciales. Une première «conférence sur les études de marché » organisée à Washington D.C. fut suivie par une série d'études importantes, qui débouchèrent sur l'organisation du recensement de la distribution de 1929. Ce dernier, en organisant pour la première fois la production d'une information générale et systématique sur les pratiques commerciales (décompte national des détaillants et des grossistes ; données sur les ventes, le nombre d'employés, les salaires, les marchandises traitées, etc.), donna une impulsion décisive techniques d'études aux et aux études de marché (construction des enquêtes, des questionnaires et des échantillons ; recherche centrées sur le consommateur), par opposition à l'orientation descriptive, économique et institutionnelle des travaux précédents (Lockley (L.C.), « Notes on the History of Marketing Research », Journal of Marketing, Vol. 14, April 1950, p. 736). 
seulement la thématique même du marketing (l'engagement du recensement fonctionnait comme reconnaissance implicite de l'importance d'une connaissance des circuits de distribution) mais aussi ses méthodes (l'usage bureaucratique des statistiques légitimait les procédures qui commençaient à se répandre dans les affaires) et ses finalités (l'État admettait l'importance, pour les milieux d'affaires, de la disponibilité d'informations factuelles générales d'après lesquelles ils puissent régler leur action ${ }^{39}$ ) — comme l'écrivent Luc Boyer et Noël Equilbey, «Le marketing avait trouvé ses lettres de noblesse dans cette crise du marché ${ }^{40}$.

Ainsi, le parallélisme marketing/New Deal se muait en convergence: les marketers entendaient bien tirer parti des rapports existant entre leur projet et celui de l'administration Roosevelt pour justifier leur action; ils pariaient sur le succès de la régulation macroéconomique pour promouvoir leur propre science de la régulation marchande. Aussi, à partir des années 1930, le développement de l'appareillage statistique d'État fut-il indissociable du formidable essor des études de marchés et des méthodologies correspondantes (mesure de corrélation, théorie de l'échantillonnage, analyse multivariée, usage de panels, etc.) ${ }^{41}$. Car la contribution de la nouvelle politique économique aux avancées du marketing n'était pas uniquement symbolique.

D'abord, la production de données fédérales fournissait d'utiles informations pour l'amélioration et le développement des techniques et des savoirs commerciaux ${ }^{42}$. Ensuite, à une époque où les entreprises ne recrutaient plus, le développement de fonctions techniques de haut niveau au sein de l'administration fédérale offrait des perspectives d'emploi alléchantes pour les jeunes cadres formés par les business schools ${ }^{43}$. Enfin et surtout, le renouvellement conceptuel de la doctrine économique ouvrait sur un changement radical de la pensée marketing. L'argument du New Deal, on l'a vu, autorisait la disqualification des savoirs et techniques anciennes, forgées pour la maîtrise d'un monde désormais révolu. Dès lors, la codification des pratiques antérieures n'était plus de mise - à quoi bon en effet répertorier des compétences inopérantes ? les choses économiques n'étant plus ce qu'elles étaient, les marketers pourraient bientôt quitter l'approche inductive de leurs débuts pour une orientation résolument déductive - construire, ex nihilo, la nouvelle science de la distribution :

39. Brown (G.H.), «What Economists Should Know About Marketing », Journal of Marketing, Vol. 16, January 1951, p 61.

40. Boyer (L.) \& Equilbey (N.), Histoire du management, Paris, Éditions d'Organisation, 1990, p. 113.

41. Bartels (R.), The History of Marketing Thought, op. cit., p. 126.

42. Converse (J.), Survey Research, United States Roots and Emergence, 1890-1960, Berkeley, University of California Press, 1987, p. 90.

43. Cochran (Th.C.), Business in American Life: A History, op. cit., p. 320. 
Dans le futur, nous pouvons espérer un très haut degré de perfection dans le développement d'une procédure scientifique en matière de recherche dont l'American Marketing Association sera certainement le moteur. Mais développer la technique n'est pas suffisant — pour important que cela soit. Ce dont nous avons le plus besoin est d'obtenir quelques principes de base qui puissent servir de guides aux processus de marketing. [...] Nous avons passé le moment où nous enseignions seulement ce qui se fait dans les affaires. Nous n'hésitons pas à critiquer le monde des affaires lorsqu'il est inefficace en matière de méthode ou antiéconomique dans ses objectifs ${ }^{44}$.

\section{De la réforme des sciences de gestion à la réforme des spécialistes du marketing}

\section{La réforme des sciences de gestion}

«Dans le futur »: à la fin de l'entre-deux-guerres, certains marketers rêvaient d'un marketing qui fût vraiment scientifique, c'est-à-dire d'un marketing qui permît d'inverser la relation historique entre savoir et pouvoir gestionnaire - de passer d'une gestion plus ou moins scientifique à une véritable science de gestion : au lieu de partir de la pratique pour constituer le savoir - au lieu d'accepter que le marketing procédât de la gestion — on espérait que le savoir pût fonder la pratique - on souhaitait faire en sorte que le marketing précédât la gestion.

Sans doute, la formulation des premiers concepts et principes de marketing, la mise au point des techniques d'études de marché, et surtout les propositions de Charles W. Hoyt et de Percival White avaient-elles ouvert la voie. Mais les premières notions ne faisaient que codifier les pratiques, les premières études restaient essentiellement descriptives et, si l'on en juge à la littérature de l'époque, l'idée pour le coup innovante d'une gestion scientifique des marchés n'avait reçu qu'un écho limité auprès des marketers universitaires.

Certes, dans les années 1950, une innovation considérable fit son apparition dans le monde commercial américain: le marketing concept. Le marketing concept proposait de subordonner la réalisation des profits à la satisfaction du consommateur, de soumettre la gestion de toute entreprise à l'observation méthodique du marché, donc de plier les autres services de gestion aux commandements du département marketing. La nouveauté résidait moins dans l'idée elle-même, qui rappelait beaucoup, par exemple, l'idée de gestion scientifique des marchés de Percival White, que dans sa reprise systématique et enthousiaste par les milieux d'affaires américains: la rhétorique conquérante du marketing concept traduisait au mieux l'émergence d'une nouvelle pratique de gestion, voire l'avènement d'une nouvelle économie fondée sur le service rendu à la clientèle ; elle témoignait, à tout le moins, 
de l'indéniable progression de la fonction et des hommes du marketing dans les entreprises américaines, et de leur influence croissante sur la conduite des affaires ${ }^{45}$.

Mais paradoxalement, l'essentiel, ici, est moins de mesurer l'éventuel effet performatif du marketing concept sur l'économie américaine que de relever son manifeste manque d'effets cognitifs sur la communauté des marketers-universitaires. Si les milieux d'affaires expérimentèrent ou vantèrent le marketing concept tout au long des années 1950, il fallut attendre le début des années 1960 pour que les universitaires du marketing s'en saisissent tout à fait. Pourquoi ce décalage ? Pourquoi les universitaires ont-ils si longtemps résisté ? Et pourquoi, finalement, ont-ils cédé au tournant des années 1960 ?

A priori, les réticences des universitaires à l'égard du marketing concept peuvent surprendre: mettre le consommateur au centre du marketing, n'était-ce pas, pour les professeurs, trouver le meilleur moyen de faire du marketing une science distincte de la pratique, de subordonner la gestion de l'entreprise à l'observation scientifique des marchés ? Oui et non. Oui, si l'on se souvient du vœu d'indépendance et de scientificité des marketers de l'avant guerre, soucieux de fonder leur discipline comme gestion de l'interface entreprisemarché. Non, si l'on se rappelle aussi l'identité particulière de ces premiers marketers, et le type de science qu'appelait la prise en compte du marketing concept. Souvenons-nous : les pionniers du marketing, formés à l'économie institutionnelle et à l'analyse descriptive des circuits de distribution pouvaient certes réaliser des études de marché, mais ils ne disposaient en aucun cas des compétences qui leur eussent permis de percer les mystères du comportement du consommateur et/ou de modéliser le fonctionnement des marchés.

Les premiers marketers n'étaient ni fous, ni aveugles : en observant le monde des affaires, ils voyaient combien le marketing concept attirait les consultants en tous genre, les Ernest Dichter, Burleigh Gardner et autres Sidney Levy, tous ceux qui proposaient les études de «motivation », c'est-à-dire la psychanalyse du consommateur comme nouvelle technique de maîtrise des marchés ${ }^{46}$. En regardant le monde des sciences sociales américaines, les pionniers du marketing voyaient combien les spécialistes des nouvelles technologies mises à l'honneur durant la guerre - la recherche opérationnelle, l'économétrie, la théorie générale des systèmes $^{47}$ - lorgnaient vers la gestion pour assurer leur reconversion dans un cadre civil, et

45 Chandler (A.D. Jr.), La main visible des managers : une analyse historique, op. cit.

46 Dichter (E.), The Strategy of Desire, Garden City, Doubleday, 1960 ; Gardner (B.) and Levy (S.J.), « The Product and the Brand», Harvard Business Review, March-April 1955, pp. 33-39; Levy (S.J.), «Symbols for Sale », Harvard Business Review, Vol. 37, No. 4, 1959, pp. 117-124.

47 Stasch (S.F.), «Operations Research: Its History, and Its Growth in Business and Industry », Northwestern University Archives, Biographical File, Stanley Stasch, Fall 1965 ; Adler (L.), «Systems Approach to Marketing », Harvard Business Review, Vol. 45, May-June1967, pp. 105-118 ; Reidenbach (E.) \& Oliva (T.A.), «General Systems Theory and the Development of Marketing Thought», in Hollander (S.C.) \& Savitt (R.) (eds.), First North American Workshop on Historical Research in Marketing, East Lansing, Michigan State 
combien une gestion des marchés fondée sur le consommateur offrait une prise favorable à l'application de ce genre de techniques. Pour rester en place, mieux valait donc faire en sorte que l'objet «consommateur» ne devînt pas le cheval de Troie des spécialistes nouveaux, mieux valait continuer à parler du marché, des produits, des institutions et des fonctions du marketing — bref : mieux valait se méfier du marketing concept.

Pourtant, en 1959, les fondations Ford et Carnegie lancèrent un important programme d'aide pour une réforme des sciences de gestion. Ce programme, piloté par Robert Gordon et James Howell, deux économistes favorables aux nouvelles sciences sociales et quantitatives, consistait à subordonner le financement des business schools d'une part à l'abandon de l'approche descriptive et inductive d'avant-guerre et d'autre part à l'adoption d'une démarche fondée sur la double mise en auvre des techniques quantitatives et des sciences du comportement ${ }^{48}$.

L'alternative, pour les anciens, était cruelle: résister aux recommandations de la fondation Ford, c'était voir sa business school d'appartenance passer à côté d'alléchantes subsides ; accepter la réforme, c'était perdre son identité d'enseignant, se faire dépasser par des approches pour lesquelles on n'avait aucune compétence. L'alternative était cruelle, mais elle n'était pas sans issue. Pour concilier l'inconciliable, pour préserver les intérêts contradictoires de leurs carrières personnelle et de leurs institutions communes, les anciens eurent l'idée de se déplacer : ils renoncèrent aux terrains de l'enseignement et de la recherche, et migrèrent vers les positions de responsables administratifs de leurs écoles. En acceptant d'organiser ce qui les dépassait, en se faisant les administrateurs de la réforme, bref, en s'appliquant à gérer l'application des techniques quantitatives et des sciences du comportement aux sciences de gestion, les vieux gestionnaires parvinrent à rester au centre des business schools tout en appliquant efficacement les orientations nouvelles ${ }^{49}$.

En marketing, la réforme des années 1960 fut d'autant mieux reprise qu'on pouvait la présenter comme un prolongement logique du marketing concept. Une fois le deuil de l'économie institutionnelle consommé, les techniques quantitatives et la psychologie du consommateur pouvaient être repris comme outils «naturels» d'une gestion fondée sur l'« orientation consommateur » — l'ère du marketing management pouvait commencer. Grâce au recrutement avisé de jeunes spécialistes des nouvelles sciences sociales, économiques et quantitatives, le marketing des années 1960 s'orienta dans une double direction : d'un côté,

University, 1983, pp. 170-180.

48. Gordon (R.A.) \& Howell (J.E.), Higher Education for Business, New York, Columbia University Press, 1959.

49. Cochoy (F.), «Quand le marketing est remis en question... dans les années 60 », Revue française de gestion, $\mathrm{n}^{\circ} 125$, septembre-octobre 1999, pp. 128-134. 
l'application de la recherche opérationnelle et de l'économétrie permit la naissance de la «marketing science », c'est-à-dire d'un courant capable de modéliser et d'optimiser le comportement des marchés; d'un autre côté, l'importation des sciences statistiques, psychologiques et sociales donna naissance à la consumer research, c'est-à-dire à une approche permettant l'étude serrée du comportement du consommateur.

À partir de cette double orientation, on put prolonger/dépasser les intuitions de Wendell R. Smith et Wroe Alderson, qui avaient proposé, dès avant la réforme, de tirer parti de la théorie économique de la concurrence imparfaite chère aux économistes de l'école de Cambridge pour clarifier/maîtriser les pratiques anciennes de segmentation et de différenciation des produits $^{50}$. Grâce aux techniques quantitatives et aux sciences du comportement, on fut en mesure de développer les concepts et les procédures nécessaires à la mise en œuvre d'un véritable marketing management — d'une gestion technique et intégrée du marché. Plus précisément, le programme de marketing management consista d'une part à proposer l'idée de marketing mix, laquelle arrime la gestion des marchés à la définition d'une combinaison optimale et contrôlée des politiques de tarification, de publicité, de conception des produits et de distribution ${ }^{51}$; ce programme consista d'autre part à compléter et renforcer le modèle du marketing mix grâce à la reprise systématique de la topique taylorienne de la planification, de l'analyse et du contrôle ${ }^{52}$.

Autour de la triade marketing concept/marketing management/marketing mix, l'application croissante des techniques statistiques au marketing permit de montrer combien, au-delà du prix, la concurrence se jouait sur l'aspect multidimensionnel du produit — c'est-àdire sur la marque, sur le service, sur l'emballage, etc. ${ }^{53}$ - et donc de jouer sur ces diverses dimensions pour configurer les marchés; l'usage des ordinateurs, des techniques économétriques et des méthodes de modélisation déboucha par ailleurs sur la mise au point de véritables boîtes noires pour une gestion technique des marchés ${ }^{54}$; les conceptualisations des sciences sociales et l'étude systématique des pratiques de consommation autorisèrent la modélisation du comportement d'achat et l'application de ces modèles à la définition des

50. Smith (W.R.), «Product Differentiation and Market Segmentation as Alternative Market Strategies », Journal of Marketing, Vol. 21, July 1956, pp. 3-8 ; Alderson (W.), Marketing Behavior and Executive Action, Homewood, Richard D. Irwin, 1957.

51 Borden (N.H.), «The Concept of the Marketing Mix », Journal of Advertising Research, June 1964, pp. $2-7$; McCarthy (E.J.), Basic marketing: A Managerial Approach, Homewood, Richard D. Irwin, 1960.

52. Kotler (Ph.), Marketing Management: Analysis, Planning and Control, Englewood Cliffs, Prentice-Hall, 1967.

53 Green (P.E.), «Bayesian Decision Theory in Pricing Strategy », Journal of Marketing, Vol. 27, January 1963, pp. 5-14.

54. Little (J.D.C.), « Decision Support Systems for Marketing Managers », Journal of Marketing, Vol. 43, Summer 1979, pp. 9-26. 
stratégies marketing ${ }^{55}$.

La réforme des années 1960 eut donc pour conséquence la mise en place rapide d'une double performation de l' «économie», au double sens que Michel Callon assigne à ce terme $^{56}$ : d'une part, la réforme performa l'économie comme discipline (economics), en la faisant migrer du macro au micro, en doublant la définition et la modélisation du circuit économique d'ensemble par un formatage et une modélisation isomorphes des pratiques gestionnaires; d'autre part, la réforme performa l'économie comme espace d'activité marchande (economy), dans la mesure où l'application des mêmes démarches, des mêmes techniques, des mêmes instruments et des mêmes procédures, depuis l'État jusqu'à l'entreprise en passant par l'ensemble des réseaux commerciaux, ne put que renforcer l'efficace d'une gestion des marchés.

Les expressions contemporaines de «management de l'État» ou de «gouvernement de l'entreprise $»^{57}$ montrent, si besoin était, combien l'économie et la gestion se sont déplacées vers la politique, combien la politique s'est repliée sur l'économie et la gestion. Au terme d'un gigantesque processus de traduction, d'alignement et de combinaison des sciences et des pratiques modernes, économie, gestion et politique ont fini par ne plus être que les différentes dimensions d'un même tissu, d'un même réseau socio-technique. D'un côté, au niveau général et public, on avait la régulation macroéconomique d'ensemble, les appareils comptables et politiques nationaux; de l'autre, au niveau local et privé, on eut désormais une régulation microéconomique gestionnaire.

Pourtant, ce que l'on a peu remarqué, c'est que l'une n'allait pas sans l'autre: si le marketing moderne, comme on l'a vu, est le fils naturel des innovations économiques et scientifiques du New Deal et de l'après-guerre, l'économie moderne doit beaucoup, pour sa pertinence, à la contribution des sciences/pratiques managériales. Seul le management empirique et microéconomique des marchés permet l'affirmation d'une «self-fulfilling economy »; ce n'est qu'à partir de la généralisation des mêmes approches, des mêmes méthodes et des mêmes outils, ce n'est que sous l'emprise du double appareillage de la politique économique et du marketing management que les pratiques marchandes contemporaines ont pu quitter définitivement l'autorégulation du marché smithien pour entrer dans le cadre régulier du contrôle politique, technique et gestionnaire.

55. Howard (J.A.) \& Sheth (J.N.), The Theory of Buyer Behaviour. New York, John Wiley \& Sons, 1969.

56. Callon (M.), «Introduction: The Embeddedness of Economic Markets in Economics », in Callon (M.) (ed.), The Laws of the Markets, Oxford, Blackwell, pp. 2-57.

57. $C f$., respectivement, Laufer (R.) \& Paradeise (C.), Le prince bureaucrate, Paris, Flammarion, 1982 ; Gomez (P.-Y.), Le gouvernement de l'entreprise. Modèles économiques de l'entreprise et pratiques de gestion, Paris, InterÉditions, 1996. 


\section{Des nouvelles formes de marketing management à la réforme des spécialistes du marketing}

Pourtant, en marketing, vers la fin des années 1960, les succès du marketing concept, du marketing management et du marketing mix avaient des conséquences inattendues. Nous l'avons souligné, les orientations nouvelles n'avaient dû leur mise en œuvre qu'au recrutement d'hommes nouveaux; le recrutement de spécialistes était le corollaire indispensable de la quantification et de la «socialisation» du marketing. Or, si les business schools entendaient favoriser l'application des sciences nouvelles aux sciences de gestion, les hommes des sciences nouvelles étaient souvent moins attachés à l'application gestionnaire de leur savoir qu'à la poursuite, au sein du marketing, des orientations de leurs disciplines d'origine.

Ainsi, lorsque certains proposèrent, dans le contexte de contestation endémique de la fin des années 1960, d'inventer un concept élargi de marketing susceptible de fonder un marketing social, c'est-à-dire un marketing applicable aux organisations sans but lucratif ${ }^{58}$, d'autres reprirent ce programme comme un appel au développement d'un marketing sociétal, c'est-à-dire un marketing plus fondamental, davantage préoccupé par l'étude du rôle social des pratiques commerciales que par la recherche de l'efficacité gestionnaire ${ }^{59}$. En quelque sorte, l'idée de marketing sociétal permit à ses adeptes non seulement de prendre une certaine distance politique vis-à-vis de l'orientation gestionnaire, mais surtout de renouer avec la relative indépendance scientifique de la psychologie, de la sociologie ou de l'économie théorique dont ils étaient issus ; le marketing social/sociétal offrit la possibilité d'une certaine désapplication de la science appliquée.

Le marketing des années 1970-1980 finit ainsi par juxtaposer d'un côté le marketing le plus technique, le plus pragmatique et le plus appliqué et d'un autre côté un marketing de plus en plus orienté vers la contestation et/ou vers la recherche fondamentale, vers l'étude du consommateur pour le consommateur, plutôt que vers l'étude du consommateur pour la gestion optimale des marchés. Que penser de cette dérive ? La réforme (au sens de libération) des spécialistes va-t-elle dans le sens d'une moindre contribution du marketing au fonctionnement de l'économie de marché ? Sans doute, le double élargissement thématique et

58 Kotler (Ph.) \& Levy (S.J.), «Broadening the Concept of Marketing », Journal of Marketing, Vol. 33, January 1969, pp. 10-15 ; Lazer (W.), «Marketing's Changing Social Relationships », Journal of Marketing, Vol. 33, January 1969, pp. 3-9 ; Kotler (Ph.) \& Zaltman (G.), « Social Marketing: An Approach to Planned Social Change », Journal of Marketing, Vol. 35, July 1971, pp. 3-12.

59. Spratlen (T.H.), «The Challenge of Humanistic Value in Marketing», in: David L. Sparks (ed.), Broadening the Concept of Marketing, Fall 1970, Chicago, American Marketing Association, p. 47 ; Sweeney (D.J.), «Marketing : Management Technology or Social Process ?» Journal of Marketing, Vol. 36, October 1972, pp. 3-10 ; Tucker (W.T.), « Future Direction in Marketing Theory », Journal of Marketing, Vol. 38, April 1974, pp. 30-35 ; Dixon (D.F.), «The Poverty of Social Marketing », MSU Business Topics, Vol. 26, Summer 1978, pp. 51-56. 
disciplinaire du marketing et la prolifération de recherches contradictoires sans intérêt ou sans préoccupations pratiques plaident-ils en faveur d'un certain relâchement des liens entre science et gestion. Pourtant, à y bien regarder, le relâchement des réseaux techniques, cognitifs et humains du marketing va davantage dans le sens de leur redéploiement que de leur rupture, et pour deux raisons.

D'abord, l'invention du marketing social a permis l'extension du marketing au-delà du cercle étroit de la seule pratique des affaires privées. L'idée de marketing social, loin de rompre avec l'ancrage gestionnaire de la discipline, a favorisé au contraire l'extension de cet ancrage à des secteurs qui, jusque-là, lui avaient échappé : l'invention du marketing politique, du marketing des causes, du marketing des affaires publiques ou du planning familial (etc.) a fini par rabattre vers la gestion marketing de l'économie de marché toute une série d'institutions qui lui étaient a priori étrangères, voire hostiles.

Ensuite, la reformulation du marketing social comme marketing sociétal a permis l'insertion des spécialistes de consumer research auprès des organes de l'État chargés de veiller à la protection du consommateur - Federal Trade Commission, Federal Drug Administration, Consumer Product Safety Commission, Office of Consumer Affairs, Office of Consumers' Education ${ }^{60}$. Le développement du consumérisme, paradoxalement, loin de condamner le marketing pour manquement à ses engagements, a plutôt reconnu son efficacité ; il a contribué à rendre les marketers toujours plus indispensables et présents dans l'économie. Grâce à la contestation consumériste, les spécialistes du marketing et leurs méthodes d'enquête furent convoqués, à titre d'experts et de corps d'expertise, pour éclairer les tribunaux lors des affaires de publicité mensongère, de fraude commerciale, de tromperies et d'abus en tous genres à l'égard du consommateur ${ }^{61}$. Les vertus comme les fautes du marketing ont ainsi contribué à généraliser la diffusion des méthodes, des concepts et des hommes de la discipline des marchés au sein de l'ensemble des institutions américaines, depuis l'entreprise privée jusqu'aux agences fédérales, en passant par l'appareil judiciaire.

Enfin, l'interprétation du marketing sociétal comme marketing «pur » ou «fondamental » aura favorisé, du moins aux États-Unis, la poursuite de la migration d'autres spécialistes ou spécialités vers le marketing, telle l'épistémologie, l'anthropologie ${ }^{62}$, l'histoire ${ }^{63}$, le

60. Bloom (P.N.) \& Greyser (S.A.), «The Maturing of Consumerism », Harvard Business Review, Vol. 59, November-December 1981, pp. 130-139.

61 Kassarjian (H.H.), «Scholarly Traditions and European Roots of American Consumer Research », in Laurent (G.), Lilien (G.L.) \& Pras (B.) (eds.), Research Traditions in Marketing, Boston, Kluwer Academic Publishers, 1994, p. 273.

62. Sherry (J.F.), «Marketing and Consumer Behavior: Windows of Opportunity for Anthropology», Journal of the Stewart Anthropological Society, Vol. 16, No. 1, 1986, pp. 60-95.

63. Fullerton (R.A.), «How Modern is Modern Marketing? Marketing's Evolution and the Myth of the "Production Era"», Journal of Marketing, Vol. 52, January 1988, pp. 108-125. 
postmodernisme $^{64}$, ou même la sémiotique et la critique littéraire ${ }^{65}$. De même que la segmentation du marketing entre marketing-pour-l'entreprise et marketing-pour-le-secteur public-et/ou-sans-but-lucratif a eu pour effet de soumettre tous les secteurs de l'Amérique à la micro-gouvernance commerciale, la segmentation du marketing entre marketing fondamental et marketing appliqué a eu pour conséquence de convoquer l'ensemble des sciences humaines et sociales autour d'une analyse systématique des marchés ${ }^{66}$.

\section{Conclusion}

En somme, l'histoire retracée nous montre que, chaque fois, le marketing est parti de l'économie pour faire de l'économie tout en faisant l'économie de l'économie! D'anciens économistes ont quitté l'économie pour observer l'économie, pour ensuite profiter de la crise économique pour reformater à leur façon le nouvel interventionnisme économique. Puis les mêmes marketers ont fini par importer les modes d'analyse et les spécialistes de la macroéconomie quantitative pour façonner des outils permettant la gestion marketing des entreprises. Chemin faisant, le marché s'est trouvé de plus en plus enserré dans un double ensemble de règles et de procédures, de concepts et d'experts, qui le définissent et le façonnent.

Sans doute, l'efficacité du marketing reste problématique ${ }^{67}$, ses performances sont difficiles à décrire, complexes à évaluer, délicates à mesurer empiriquement. Mais la généralisation sociale du marketing ne fait guère de doute. Les managers en passent par le marketing, les marketers passent par le management. L'histoire montre que les uns et les autres font certes ce qu'ils veulent du marketing et de la gestion, que les uns comme les autres peuvent les dépasser ou s'en passer, mais la gestion marketing, pour chacun, constitue bien une référence, un horizon de l'action, dont nous finissons toujours par retrouver les traces distordues, ambiguës, multivalentes mais toujours présentes et significatives dans l'ensemble des manifestations contemporaines du capitalisme américain, depuis les numéros d'appel gratuits jusqu'aux multiples enquêtes de consommation, en passant par la responsabilité des marques, la culture consumériste, et surtout l'extrême généralisation du vocabulaire de la discipline, l'usage constant, par l'ensemble des acteurs sociaux et dans toutes les situations,

64. Firat (A.F.), « The Consumer in Postmodernity », in Holman (R.H.) \& Solomon (M.R.) (eds.), Advances in Consumer Research, Vol. 18, 1991, pp. 70-76.

65. Stern (B.B.), «Humanizing Marketing Theory: Literary Criticism and the Art of Marketing », in Lichtental (D.) \& al. (eds.), Marketing Theory and Application, 1990 Winter Educator's Conference, Chicago, American Marketing Association, 1990, pp. 24-28; Holbrook (M.B.) \& Hirschman (E.C.), The Semiotics of Consumption, Berlin, Mouton de Gruyter, 1992.

66. Pour un exposé plus précis, $c f$. Cochoy (F.), Une histoire du marketing, Paris, La Découverte, 1999.

67 Marion (G.), «Le marketing management en question », Revue Française de Gestion, janvier-février 1995, pp. 15-30. 
des métaphores de la segmentation, du positionnement, de la publicité, des cibles, des niches (etc.).

La meilleure preuve de la performance économique du marketing se trouve peut-être non pas dans l'observation de son incidence directe sur le marché, mais plutôt dans le constat de son impact indirect auprès des économistes : ces derniers, depuis l'École de Cambridge, théorisent la concurrence imparfaite, elle-même parfaitement gérée par le marketing. Aujourd'hui, économistes et sociologues réfléchissent aux modalités de la «concurrence hors prix » et de l' «économie du signal », à «l'économie de la ou des qualités », aux diverses «conventions » qui instrumentent la définition des marchés et la qualification des produits ${ }^{68}$. Or, qu'a fait le marketing, bien avant les économistes et les sociologues, sinon fournir aux offreurs et aux demandeurs les conventions qui leur permettent de qualifier les produits, qu'a fait le marketing, sinon mettre au point les outils susceptibles de desserrer la contrainte des prix pour définir au mieux la qualité des produits ? Nous-mêmes, analystes contemporains de l'économie de marché, courons souvent, sans le savoir, après la science des marketers. Si l'économie fut, à l'origine, la muse du marketing, il se pourrait bien que le marketing soit devenu, au fil du temps, la ruse de l'économie.

68. Spence (M.), «Job Market Signaling», The Quarterly Journal of Economics, Vol. 87, 1983, pp. 355-374 ; Eymard-Duvernay (F.), «Conventions de qualité et formes de coordination », Revue Économique, L'économie des conventions, Vol. 40, n² 2, mars 1989, pp. 329-359 ; Karpik (L.), «L'économie de la qualité », Revue Française de Sociologie, Vol. 30, n² 2, avril-juin, pp. 187-210 ; Callon (M.), «L'économie des qualités », Politix, à paraître. 